\title{
Uncertainty Visualization and Hole Filling for Geometric Models of Ancient Water Systems
}

\author{
Jeffrey Forrester ${ }^{1}$, William McVicker ${ }^{1}$, Timmy Gambin ${ }^{2}$, Christopher Clark ${ }^{3}$ and Zoë J. Wood ${ }^{1}$ \\ ${ }^{1}$ California Polytechnic State University, San Luis Obispo, CA, USA \\ ${ }^{2}$ University of Malta, Malta \\ ${ }^{3}$ Harvey Mudd College, Claremont, CA, USA \\ $\{$ jdforres, zwood $\} @$ calpoly.edu
}

Keywords: Surface reconstruction, Geometric modeling, Hole filling, Uncertainty visualization.

Abstract: Geometric data acquired via a scanning process can suffer from holes due to errors in the acquisition process, noise, or challenges in merging multiple inputs together into a unified map. We present a straight forward algorithm to fill holes in incomplete evidence grids representing acquired geometric data. We also present our methods to apply learning in order to statistically evaluate the proposed hole filling algorithm. This analysis validates our proposed method for hole filling and additionally enables the construction of a probability distribution function to represent the accuracy of the filled data per model. During surface reconstruction, this function can be used to visualize the certainty of the filled geometry via transparency and coloring giving the user an understanding of the data's accuracy. This work is motivated by a multi-year project to construct educational visualizations of ancient water storage systems, i.e. cisterns and wells within churches, fortresses and homes on the islands of Malta, Gozo and Sicily.

\section{INTRODUCTION}

Geometric data acquired via sensors and scanners often suffers from missing data due to noise, acquisition error, and geometric complexities. Mapped objects are generally closed surfaces and the representative data is considered incomplete until the surface is closed. Surface reconstruction and hole filling are a well studied problem, however challenges remain for noisy data with significant gaps. We present an algorithm for hole filling acquired data with large gaps, along with our methods to statistically evaluate this algorithm and calculate an estimate of the filled holes accuracy. During the statistical analysis stage of the algorithm, a functional representation of certainty is built. This functional representation can be seen as a probability distribution function $(p d f)$. Once surface reconstruction and hole filling are completed, the $p d f s$ are incorporated in the final visualizations of constructed models to illustrate the uncertainty of filled regions.

This project is a part of a larger one, specifically aimed at mapping and modeling ancient water storage systems, i.e. cisterns, wells and water galleries located in most houses, churches, and fortresses of the islands of Malta, Gozo, and Sicily. Archaeologists looking to study and document ancient water systems have found the task can be difficult, dangerous, and expensive. The data used in this paper was gathered through a series of underwater robot deployments in which multiple sonar scans were gathered, then fused into a map of the scene via SLAM algorithms (Simultaneous Localization and Mapping). Such maps, which are typically evidence grids of probability values, can be treated as implicit volumes. Surfaces can be extracted from these volumes via marching cubes and then visualized. The input data for this project includes both holes and noise (see Figure 7). In this paper, we present our hole filling algorithm, including statistical analysis of the certainty of filled regions and demonstrate resulting final visualizations of the water systems.

\section{RELATED WORK}

Surface Reconstruction and Hole Filling: In this research we aim to create a tool for assisting archaeologists in their examination of underwater structures. Surface reconstruction in the underwater setting is a relatively new area of research with initial work com- 

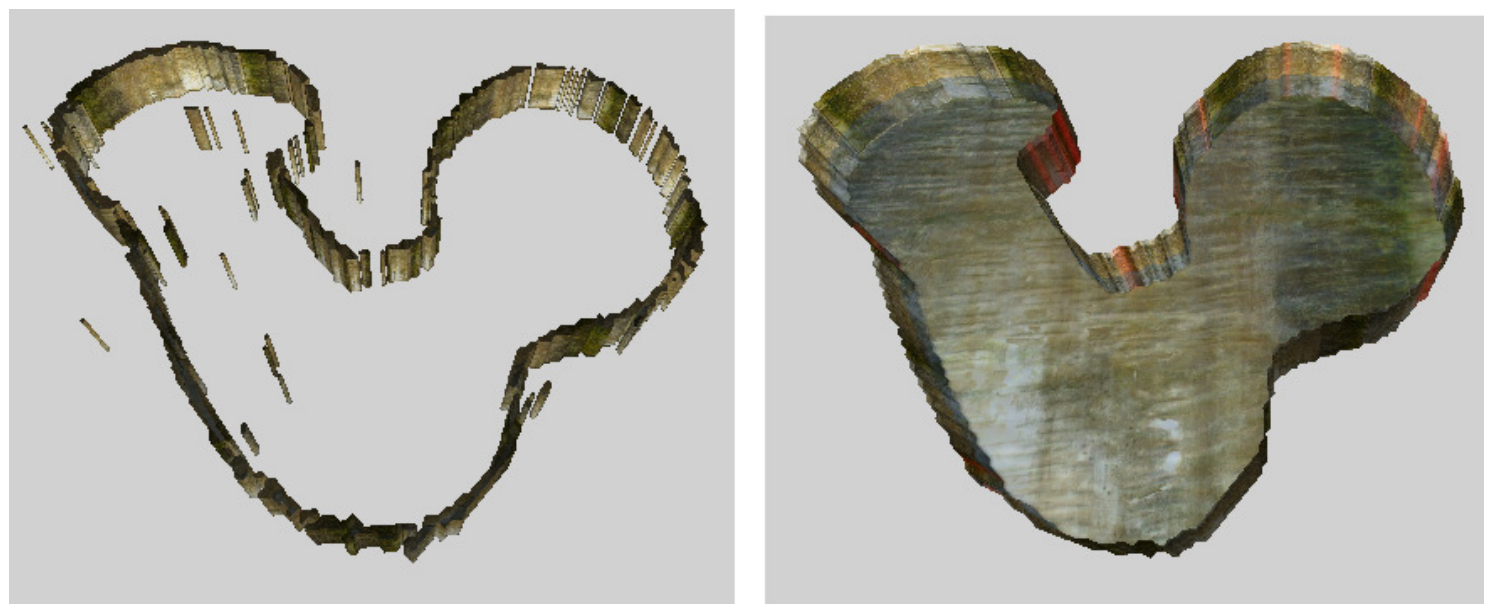

Figure 1: Geometric model extracted from an evidence grid created from sonar data. The model on the left is extracted without application of our algorithm, while the model on the right has hole filling, $p d f$ creation and uncertainty visualization applied (red regions are less certain). This cistern is in a private home in Mdina, Malta

pleted in the maritime setting (Pizarro et al., 2009) and recent work in cenotes in Mexico (Fairfield et al., 2010). Similar to the work to map cenotes (Fairfield et al., 2010), this project involves the application of marching cubes (Lorensen and Cline, 1987) to reconstruct a geometric model of underwater data, however, the cenote project relies on a very sophisticated and significantly larger more expensive ROV and sensors that would be impractical in water systems explored in this project. Our research requires mapping in tunnels with passages and access points that are relatively small (e.g. $0.30 \mathrm{~m}$ diameter at some points), requiring a micro ROV with low payload capacity and minimal sensors (i.e. scanning sonar, depth sensor, and compass).

Managing holes in acquired data is a mature field with numerous research projects addressing hole filling in both the surface and volumetric setting. For an overview of many of the relevant research projects related to polygonal repair, see the course notes on 'Geometric Modeling Based on Polyongal Meshes' (Botsch et al., 2007). Recent work in surfaces has been conducted (Bac et al., 2008), while seminal work in the volume setting includes, VRIP (Curless and Levoy, 1996) and subsequently volume diffusion (Davis et al., 2002), along with recent work in the volume setting by (Janaszewski et al., 2010). While there has been excellent work in the field of hole filling, the work presented in this paper addresses the unique challenge of acquired data from noisy sonar along with the challenge of showing information regarding confidence in our filled geometric models.
Visualization and Uncertainty: For this project, we use well established visualization techniques to create visually appealing models and to convey uncertainty (Pang et al., 1997; Schmidt et al., 2004). Uncertainty visualization are relevant to many fields (Pang et al., 1997; Schmidt et al., 2004; Pfaffelmoser et al., 2011; Grigoryan, 2002). The work presented in this paper contributes a method to statistically analyze and quantify the certainty of filled regions that could then be visualized with various uncertainty techniques.

Mapping via Underwater Robot Systems: This project relies on data acquired from algorithms for mapping with underwater robots, in particular, under water Simultaneous Localization and Mapping (SLAM) (e.g. (Williams et al., 2000), (Hernàndez et al., 2009), and (Fairfield et al., 2006)). The work of Thurn (Thrun et al., 2005) includes a good survey of the core techniques capable of fusing data from multiple sensors to create maps.

\section{ALGORITHMS AND PRACTICE}

This work is focused on reconstructing geometric models from evidence maps representing Mediterranean water storage systems (cisterns, wells and water galleries). Specifically, the space being mapped is discretized into a two dimensional grid of cells with given a likelihood $p_{i, j} \in[0,1]$ of being occupied (Thrun et al., 2005) and (White et al., 2010). The evidence grid is extrapolated into three dimensions to the appropriate height of the water system, and can then be treated as volume data, and geometric models of the scanned data can be constructed via marching 
cubes, similar to the work presented in (Forney et al., 2011). Any cell with $p_{i, j}>t$ is considered an occupied cell and associated with a wall in the model. The threshold value, $t$, is used to define occupancy and is generally set to values in the range $.65-.85$. We refer to cells in the grid at location $(i, j)$ as $x_{i j}$, and the probability of that cell, $p\left(x_{i j}\right)$, as $p_{i j}$.

Due to the acquisition process, gaps in the evidence grid are common and can lead to unwanted holes in the reconstructed models. See Figures 4 and 2, for examples of incomplete evidence grids that lead to geometric models with many holes, like those seen in Figures 1 and 6. As the acquired data is known to represent water tight structures, hole filling is necessary to construct more realistic models.

\subsection{Hole Filling}

In summary, for our setting, hole filling is achieved by fitting a function, $F$, to the $\{x, y\}$ position of a subset of occupied cells surrounding identified holes. Next, unfilled cells of the grid in the 'hole' that are crossed by $F$ are set to occupied in order to fill the hole. To visualize the effectiveness of our fitting method, we analyze the accuracy of filled regions. For each model, we execute a learning process on real data to compute a $p d f$ to represent the likelihood of the filled cell's certainty as a function of neighboring cells being filled and the distance from those cells.

\subsubsection{Simulating holes for learning}

Using acquired evidence grids, we wish to analyze our hole filling on real data. We start by identifying fairly complete sections of input data, representing walls of the water system. These walls are represented by long runs of connected occupied cells, into which we introduce 'simulated holes'. Specifically, varying length valid segments of the evidence grid are selected. A segment is a group of cells containing all neighboring occupied cells. A neighboring cell is a cell $x_{i \pm 1 j \pm 1}$. Valid segments have a minimum length, $l_{\text {min }}$, (in practice, $l_{\text {min }}=9$ )

We create 'simulated holes' by knocking out the middle section of valid segments by setting some of the occupied cells in the segment to unoccupied. When creating holes, we randomly choose segment lengths to create a variety of hole sizes. This method restricts our holes to a minimum size of around 3 cells, but allows them to be as large as a third of the longest segment length. For the removed sections, the occupied cells closest to the edge of the hole, one from each side, are defined as endpoints, $e_{i j}^{a}$ and $e_{i j}^{b}$. Endpoint detection and matching in practice is discussed in Section 3.2.

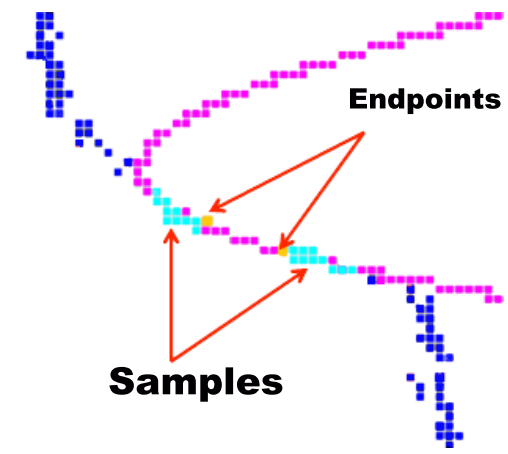

Figure 2: Definitions are shown in this figure from data from the same cistern shown in Figure 1. Occupied cells are shown in dark blue. Sample Points, $S_{S}$ are shown highlighted in light blue, endpoints are shown in orange and pink samples, $S_{F}$, correspond to a cubic polynomial fit to the hole between the two endpoints. Only the sample points, $S_{H}$ that lay between the two endpoints will be filled.

To fill each hole and measure the accuracy of each fit, we define the following (see Figure 2):

1. The shortest distance between a cell, $x_{i j}$ and a point on the fitted curve $F, f_{x y}$ as, $\left\|x_{i j}-f_{x y}\right\|$ (for higher order polynomials, this distance represents the arc length along the curve).

2. $S_{S}$ is the set of occupied cells that were not removed from the segment

3. $F$ is the polynomial fit computed using GaussJordan elimination on set $S_{S}$

4. $S_{F}$ is the set of cells in the evidence grid that lay on the fitted curve $F$

$$
\begin{array}{r}
S_{F}=\left\{x_{i j}|| x_{i}-f_{x} \mid<0.5,\right. \\
\left.\left|x_{j}-f_{y}\right|<0.5\right\}
\end{array}
$$

5. $S_{H}$ is the subset of cells belonging to $S_{F}$ that lie between the endpoints, $e_{i}^{a}$ and $e_{i}^{b}$

$$
\begin{array}{r}
S_{H}=\left\{x_{i j} \mid S_{F},\right. \\
x_{i} \leq \max \left(e_{i}^{a}, e_{i}^{b}\right), \\
x_{i} \geq \min \left(e_{i}^{a}, e_{i}^{b}\right), \\
\left.p_{i j}<t\right\}
\end{array}
$$

Given these definitions, in order to fill the 'simulated hole', the unoccupied cells from $S_{H}$ are set to filled: $\forall x_{i j} \in S_{H}, p_{i j}=1$.

All observed water storage systems contain relatively smooth man-made walls, thus we found the use of varying order polynomials (linear, quadratic, or cubic) worked well (documented shortly), however, our algorithm does not depend on the structure or fitting method of this function and any standard approach to function approximation can be used. 


\subsubsection{Probability Distribution Function}

Ideally, we would like to model the uncertainty of all the filled cells in $S_{H}$ that we have introduced during hole filling. To accomplish this, we can repeatedly create simulated holes, then fill them and measure their accuracy against the original data. This process allows us to compute a probability distribution function, $\eta\left(d_{e}\right)$, that models the likelihood that any cell $x_{i j} \in S_{F}$ has $p_{i j}>t$, as a function of the distance $d_{e}$ from $x_{i j}$ to the nearest endpoint. That is, the pdf represents the likelihood that $F$ sets the appropriate cells to being occupied, depending on the distance from the cell to the start of the hole.

$$
\eta\left(d_{e}\right)=P\left(p_{i j}^{\prime}>t \mid d_{e}, x_{i j} \in S_{H}, p_{i j}>t\right)
$$

To compute this pdf, we learn from the evidence grids by tabulating a histogram of cells of the filled region that match the original data. We analyze each fit by comparing the newly filled data with the 'known' real data. Specifically, for each cell $x_{i j} \in S_{H}$, we check to see if the cell from the original evidence grid also had an occupied cell at the same $x_{i j}$. This data is tabulated into discrete bins, based on their distance along $F$ to the nearest endpoint to build up a histogram. Specifically, for each unit along $F$, if the cell $x_{i j}$ corresponds with an occupied cell in the original data, we consider this point a hit, if not it is a miss. Distances along $F$, denoted as $d_{e}$, are measured along the arc of the fit from the nearest end point. For each type of fit: linear, quadratic, and cubic, hits and misses are tallied into discrete bins for each order polynomial.

We build up the histogram data by iteratively repeating hole filling of simulated holes for each dataset per fit, and computing hits for each hole. Once the samples have been collected, the percentage of hits at given distances along the curves are computed for each fit. See Figure 3 for an example of the discrete binned distance values of a completed histogram for one evidence grid for a quadratic fit. To create a continuous pdf for each model, the discrete binned hit percentages at distances along the curve are fit with a cubic polynomial.

The goal of this project is to reconstruct models that will be used to illustrate the shape of water systems, and it is important to distinguish between the portions of the model reconstructed from acquired data and the geometry introduced during the hole filling, as well as the accuracy of the filled data. We use each $p d f$ in order to convey this information.

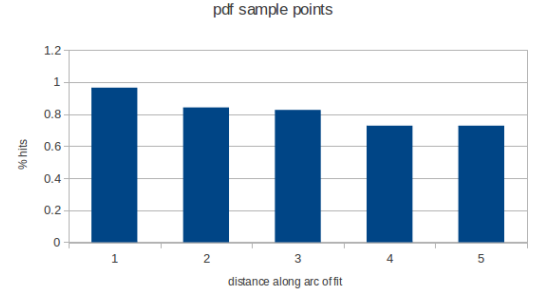

Figure 3: A histogram of tallied hits for a quadratic fit for the Tas Silg evidence grid. Bins are discretized at arc lengths zero to 5 along $F$ (measured from either end point, so the fewest hits are in the middle of the hole at a largest distance of 5 units from either endpoint)).

\subsubsection{Application of pdf Data for Visualization}

Once the $p d f$ information has been computed for a given model, the data can be applied to cells in the hole filling process. When filling a hole, each filled cell is assigned a probability based on it's distance from the closest endpoint, $d_{e}$. The $p d f$ for the specific order polynomial chosen as the best fit is referenced for certainty information and that data is assigned to the newly filled cell. We describe the error metric used for selecting the best fit in Section 3.2. Thus, every newly filled cell is given a value, $\gamma$, which represents the predicted likely-evidence of that fit matching the input model's learned shape (or actual data).

We allow the user to visualize this uncertainty by changing the coloring of the surface polygons for those extracted from evidence cells with a value of $\gamma$. For each vertex, red is added to the texture coloring based on $1-\gamma$, leading to less certain regions having higher red colorings. Figures 1, 4, 7, and 6 all show examples of the uncertainty visualizations generated with our visualization system.

\subsubsection{Validation}

During this learning process, we also validate our hole filling method overall. Specifically, we can tally overall the percentage of true positives, that is occupied cells that we would fill using our method that were originally occupied in the input data. We can also compute false positives, cells that were unoccupied in the original data but are filled via our hole filling. See Table 1 for results of the statistical analysis for seven models evaluating the results of hole filling. Note that all models perform very well with an average of $83 \%$ true positives and only $17 \%$ false positives. Overall for all data sets the computed true positives are $>68 \%$, with the most challenging data being a very long water gallery with over 87 holes.

We have presented our method to statistically analyze our hole filling method and compute a pdf which 
Table 1: Statistics for models

\begin{tabular}{c|c|c|c} 
Model name & $\begin{array}{c}\text { size } \\
\text { of grid }\end{array}$ & positives & $\begin{array}{c}\text { false } \\
\text { positives }\end{array}$ \\
\hline Case Cutietta & $120 * 120 * 26$ & $94 \%$ & $06 \%$ \\
\hline College Garden & $100 * 320 * 30$ & $89 \%$ & $11 \%$ \\
\hline Gatto Pardo & $132 * 109 * 25$ & $75 \%$ & $25 \%$ \\
\hline Keyhole & $150 * 100 * 30$ & $87 \%$ & $13 \%$ \\
\hline Site 8 & $160 * 120 * 25$ & $85 \%$ & $15 \%$ \\
\hline Tas Silg & $187 * 175 * 26$ & $83 \%$ & $17 \%$ \\
\hline Qanat & $562 * 162 * 26$ & $68 \%$ & $32 \%$
\end{tabular}

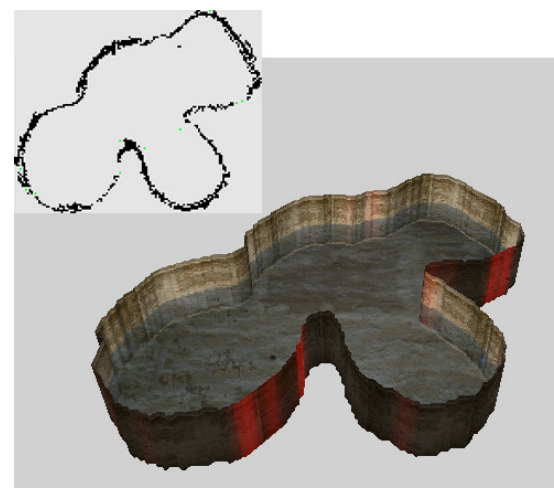

Figure 4: The evidence grid and geometric model of the Gatto Pardo cistern (private home in Mdina, Malta).

models the certainty of our hole filling, computed via a learning process. We now describe how to apply our hole filling to real holes in acquired data.

\subsection{Hole Filling in Practice}

For this expedition, investigators deployed a VideoRay ROV equipped with an underwater micron scanning sonar, depth sensor, and two video cameras. The ROV was lowered into cistern access points until it was submerged. The investigators then tele-operated the robot to navigate the underwater environment. Due to the importance of acquiring accurate data, stationary sonar scans were taken using a SeaSprite scanning sonar mounted on top of the ROV. These sonar measurements were used to generate evidence grids that in turn, were used to generate geometric models of the cisterns (McVicker et al., 2012) and (White et al., 2010), (Forney et al., 2011).

For real world acquired data, a number of subtle challenges arise when applying hole filling to construct a complete water tight model. In practice, we find two types of holes that must be filled: those due to under-sampling, characterized by very small neighboring segments and those due to missing data, characterized by larger holes between defined segments. See Figures 2 and 7 for examples of both. We choose to fill small holes first to build up information about the shape of a model, thus we fill holes in an iterative fashion with the smallest holes being filled first via a distance threshold, $E_{\tau}$, that is expanded only after all holes within that range are filled. Our algorithm for filling holes involves the following steps:

for $E_{\tau}=\min ; E_{\tau}<H ; E_{\tau}++$

Detect endpoints of valid segments

Match best pairs of endpoints

for any pairs with a size $<E_{\tau}$ fill identified holes

Endpoint Detection The first stage in hole filling is identifying the segments in the evidence grid. The evidence grid created from the sonar data may contain segments of varying thickness due to the reflection of the sonar's wide cone shaped beam off of organically shaped walls. When identifying the endpoints of segments, we cannot make assumptions about the segment's cell's connectivity or endpoint locations (See Figure 2). In order to compute approximate segment endpoints, we identify the start and end cells of long sequences or paths of occupied cells using two passes of Dijkstra's algorithm. We have found two passes of Dijkstra's to suffice in practice to identify two reasonable extremas of the segment to use as potential endpoints. The algorithm proceeds as follows:

1. Using any occupied cell $x_{i j}$ as the starting point, a Dijkstra's search traverses the neighborhood of grid cells, $x_{i \pm 1, j \pm 1}$, connecting to any unvisited occupied cell. The cell in the resulting graph which is reached via the longest path is identified as the first endpoint of the segment, $e_{i j}^{1}$.

2. A second pass is run using this endpoint, $e_{i j}^{1}$, as the root node. The node at the end of the longest path in this graph is identified as the second endpoint of the segment, $e_{i j}^{2}$.

Endpoint detection proceeds until all occupied cells in the evidence grid have been traversed. This method may miss extrema for segments that branch, however, it has been found to work well in practice.

To account for noise in the evidence grid, a segment is considered a valid segment only if the distance from endpoint to endpoint along its Dijkstra's graph is $\geq 4$, and a valid endpoint is any endpoint belonging to a valid segment. We refer to the length along a Dijkstra's graph from cell $a$ to cell $b$ as $D_{d}(a, b)$ where the cost of traversing to a neighboring occupied cell $\left(x_{i \pm 1, j \pm 1}\right)$ is always 1 . Once all valid endpoints have been identified, we are ready to identify holes as the cells with $p_{i j}<t$ that lie between pairs of valid endpoint. In order to fill these 'holes' (regions that are unoccupied) properly, corresponding endpoints must be identified and matched. 


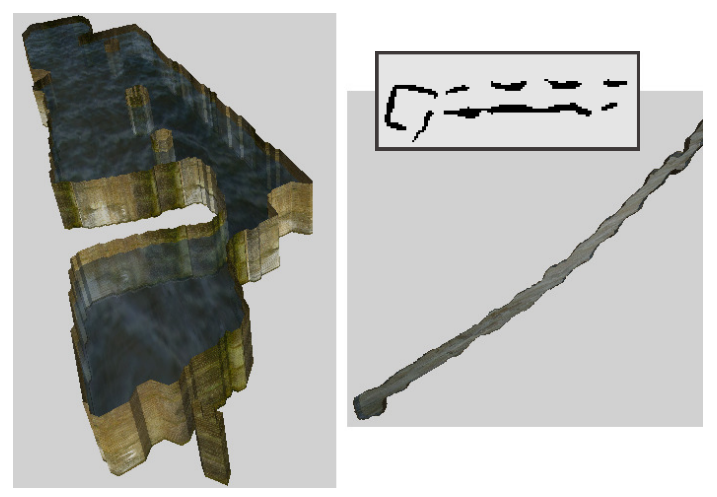

Figure 5: Left: The complete geometric model of the College Garden water system, including the correct reconstruction of three columns found in the cistern. Right: Evidence grid and geometric model of Conca D'Oro Qanat

Endpoint Matching In practice, we do not know which pairs of endpoints correspond to a hole and matching endpoint pairs that are closest together is not always accurate. Thus, given the collection of endpoints, holes are identified via the use of pairwise testing, using two criteria: distance of endpoints to one another, and how well a given pair will match the shape of neighboring segments. First, for each iteration through the hole filling algorithm, endpoint matching is constrained to only potentially create matches from any pair of endpoints, $e_{i j}^{a}$ and $e_{i j}^{b}$ whose Euclidean distance $\left(\left\|e_{i j}^{a}-e_{i j}^{b}\right\|\right)<E_{\tau}$.

Once the set of potential matches has been identified, the algorithm measures how well the fit of a potential pair's samples conforms to the local shape of their segments. For each endpoint, all cells (from the Dijkstra's path) within a threshold distance, $\delta$, are identified as the endpoint's sample points. The two endpoint's sample points are then fit with a polynomial, $F$, (as in Section 3.1.1). The best matched fit is the one with the smallest average distance from the sample points to closest points on the fit, $F$.

In order to measure the best matched fit, $S_{S}$ is defined in practice as the set of all cells of the evidence grid that fall within a given distance, $\delta$, of each endpoint, $e_{i j}^{a}$ and $e_{i j}^{b}$. That is:

$$
\begin{array}{r}
S_{S}=\left\{x_{i j} \mid p_{i j}>t,\right. \\
\left.D_{d}\left(x_{i j}, e_{i j}^{a}\right)<\delta \vee D_{d}\left(x_{i j}, e_{i j}^{b}\right)<\delta\right\}
\end{array}
$$

Given these definitions, the shape matching error is computed as:

$$
\text { Min_Err }=\frac{\sum_{x_{i j} \in S_{S}}\left(\left\|x_{i j}-f_{x y}\right\|\right)}{\left|S_{S}\right|}
$$

Once all possible sets of endpoints have been added to the list, we choose the matched endpoints with the lowest error allowing each individual endpoint to be used only once. This matching process allows us to identify the most likely endpoints that correspond to a hole in the data.

Filling Once best pairs of endpoints surrounding holes have been identified, unoccupied cells within holes are set to occupied by fitting the function $F$ to the sample data, $S_{S}$, (defined above), for the best pair. The order of $F$ is chosen to minimize Min_Err, (defined above) and hole filling is applied as defined in Section 3.1.1. That is, the unoccupied cells from $S_{H}$ are set to filled: $\forall x_{i j} \in S_{H}, p_{i j}=1$.

Because $F$ is determined by generating a function that matches local geometry, there must be welldefined local geometry available in order to fill large holes. Thus, we fill smaller holes first in order to build up long segments in the evidence grid iteratively.

Final Visualizations Once hole filling is complete, the $2 \mathrm{D}$ evidence grid is extruded into 3D. The appropriate extrusion height is defined via measured data from each specific water system site. The walls of the water systems are well modeled by the evidence grid, but in the surface creation stage a floor is added after hole filling by flood filling the interior of the now closed model and adding an empty layer to the evidence grid below the floor level. To remove small excess surface components due to noise (such as those seen in Figure 1), volume smoothing is also applied before surface extraction.

\section{RESULTS}

We have created geometric models for a dozen individual ancient water systems from the islands of Malta, Gozo and Sicily. Each of these sites suffered from incomplete data due to inaccuracies in the sonar and mapping. These inaccuracies resulted in evidence grids with numerous holes leading to extracted surfaces with holes. In this paper, we present a robust hole filling algorithm, which fills in missing data in the evidence grid, while honoring the shape of existing data. This hole filling algorithm results in water tight meshes with boundary. We present the statistical analysis used to validate this algorithm and to construct a probability distribution function to model the accuracy of the filled data. We also present details of applying our hole filling method in practice. Using the data gathered in our statistical analysis we also present uncertainty visualizations of the filled surface data. We believe this is the first such complete system for mapping and reconstructing underwater geometric 

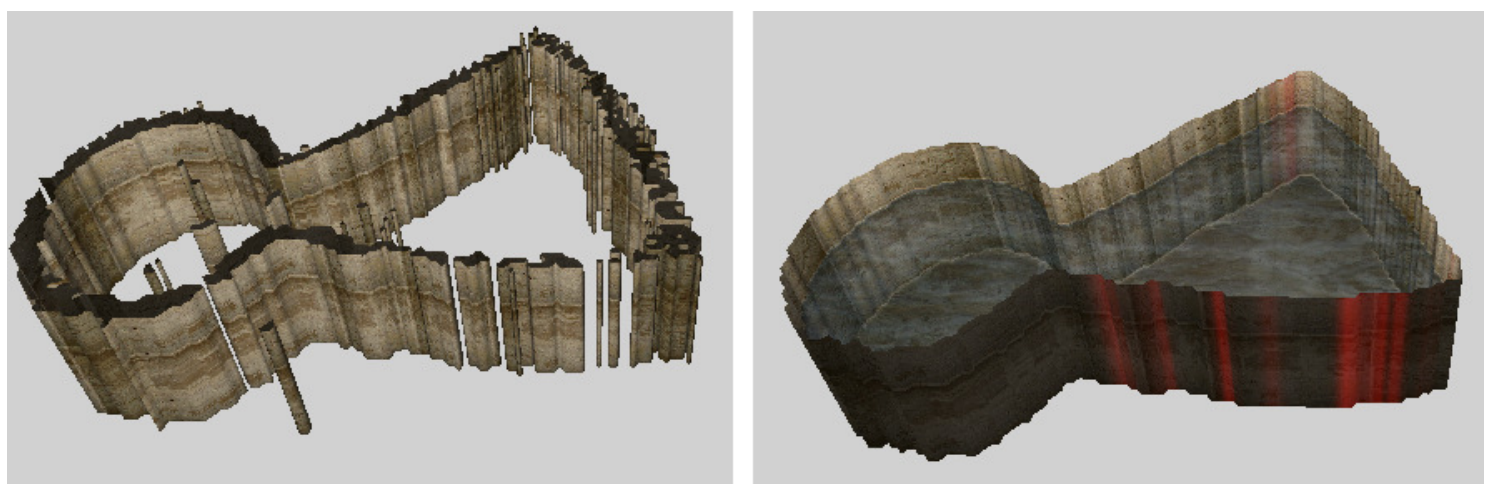

Figure 6: On the left is the geometric model of the House Dar T'ana (private home on Gozo) cistern before hole filling and on the right is the complete geometric model including uncertainty visualization of surfaces created via hole filling.

structures with hole filling and uncertainty visualizations from evidence grids.

We show surface reconstruction results and uncertainty visualization for six different ancient water systems, (the physical scale of the grids in this paper range from $0.03 \mathrm{~m}$ to $0.1 \mathrm{~m}$ per cell):

- Figure 1, shows the cistern located in a private home on Gozo, (with 18 filled holes).

- Figure 4, shows the cistern located in a private home in Mdina, Malta, (with 17 filled holes).

- Figure 7 shows results from sonar scans of the water system at Tas Silg (Malta). This ancient water system, located at the 2,000 year old temple site, was over 15 meters long and very complex with a loop structure connecting two entry points via two divergent channels, (with 42 filled holes).

- Figure 5 is the cistern at Wignacourt Museum College Garden (Malta). The cistern is fairly complex and includes several chambers and three pillars visible in the model, (with 20 filled holes).

- Figure 6 shows the cistern located at House Dar Ta'Anna - Upper courtyard, Gozo, which is a keyhole shaped cistern, (with 9 filled holes).

- Figure 5 shows the qanat at the hotel Conca d'Oro (Sicily). This very long water gallery (over 40 meters long) was a part of the historic paper factory and data collection included over 20 stationary sonar scans (with 87 filled holes).

Table 1 includes information on the size of datasets used for this work and information about the accuracy of our hole filling data for each model. Note that all datasets include numerous holes, up to 87 for the qanat in the hotel Conca d'Oro.
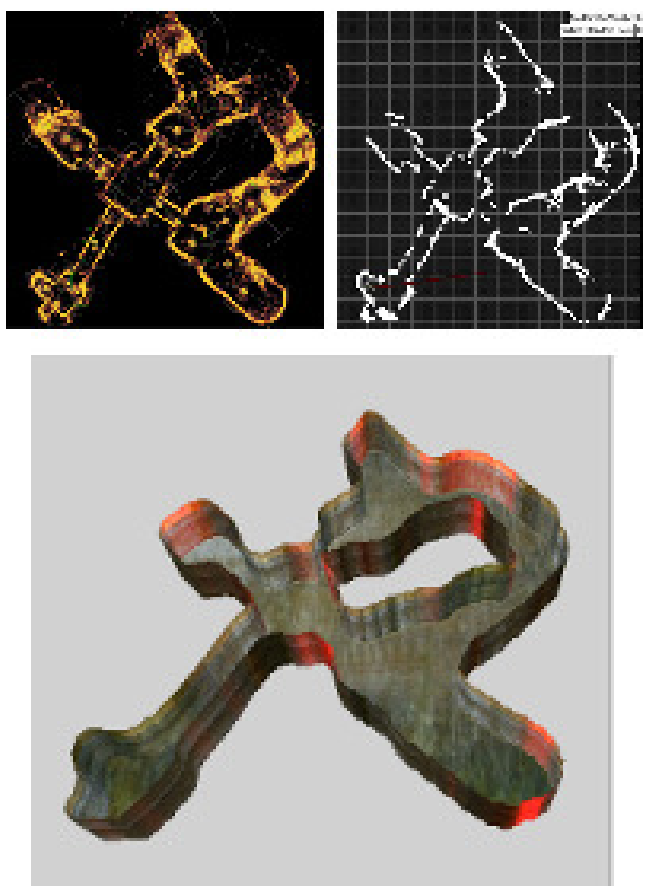

Figure 7: The top row shows both the sonar data and the evidence grid for Tas Silg. This very large water system located on an ancient temple site was over 15 meters long. The bottom image shows the complete geometric model, including uncertainty visualization of filled holes.

\subsection{CONCLUSION}

We have presented a robust straight-forward hole filling algorithm, including statistical analysis of the certainty of filled regions and demonstrate resulting final visualizations of the water systems. The hole filling performs very well even with very noisy input data and missing segments, while still preserving the original shape of the input data (such as the small connecting tunnel in Tas Silg, (Figure 7).

Future improvements to this work, include prop- 
agating probability errors from the mapping process into the evidence grid to visualize the certainty of the acquired data itself, including, incorporating a model of the sonar uncertainty more completely throughout the reconstruction process as done in (Pandey et al., 2007). Finally, some of the water systems explored contain interesting shape variation in their vertical extent and 3D mapping is an active area of our work.

\section{APPENDIX}

We wish to especially thank Christina Forney, Erik Nelson, Jane Lehr and the ICEX teams (2011 \& 2012). This material is based upon work supported by the National Science Foundation, Grant No. 0966608.

\section{REFERENCES}

Bac, A., Tran, N.-V., and Daniel, M. (2008). A multistep approach to restoration of locally undersampled meshes. In GMP'08, pages 272-289.

Botsch, M., Pauly, M., Kobbelt, L., Alliez, P., Lévy, B., Bischoff, S., and Rössl, C. (2007). Geometric modeling based on polygonal meshes. In $A C M$ SIGGRAPH 2007 courses. ACM.

Curless, B. and Levoy, M. (1996). A volumetric method for building complex models from range images. In SIGGRAPH '96, ACM Press.

Davis, J., Marschner, S., Garr, M., and Levoy, M. (2002). Filling holes in complex surfaces using volumetric diffusion. In Symposium on 3D Data Processing, Visualization, and Transmission.

Fairfield, N., Kantor, G., Jonak, D., and Wettergreen, D. (2010). Autonomous exploration and mapping of flooded sinkholes. Int'l. J. Rob. Res., 29(6):748-774.

Fairfield, N., Kantor, G., and Wettergreen, D. (2006)). Real-time SLAM with octree evidence grids for exploration in underwater tunnels. In Journal of Field Robotics, Vol 24, Issue 1-2, pp. 03-21.

Forney, C., Forrester, J., Bagley, B., McVicker, W., White, J., Smith, T., Batryn, J., Gonzalez, A., Lehr, J., Gambin, T., Clark, C., and Wood, Z. (2011). Surface reconstruction of Maltese cisterns using ROV sonar data for archeological study. In Proceedings of the 7th Int't. conference on Advances in visual computing.

Grigoryan, G. (2002). Probabilistic surfaces: Point based primitives to show surface uncertainty. In Proceedings Visualization 2002.
Hernàndez, E., Ridao, P., Ribas, D., and Batlle, J. (2009). MSISPIC: A probabilistic scan matching algorithm using a mechanical scanned imaging sonar. In Journal of Physical Agents 3:311.

Janaszewski, M., Couprie, M., and Babout, L. (2010). Hole filling in $3 \mathrm{D}$ volumetric objects. Pattern Recogn., 43(10):3548-3559.

Lorensen, W. E. and Cline, H. E. (1987). Marching cubes: A high resolution 3D surface construction algorithm. In SIGGRAPH '87, pages 163-169.

McVicker, W., Forrester, J., Gambin, T., Lehr, J., Wood, Z., and Clark, C. (2012). Mapping of ancient water storage systems with an ROV for visualization: An approach based on fusing stationary scans within a particle filter. In IEEE ROBIO.

Pandey, A. K., Krishna, K. M., and Nath, M. (2007). Feature based occupancy grid maps for sonar based safe-mapping. In Proceedings of the 20th Int'l. joint conference on Artifical intelligence.

Pang, A. T., Wittenbrink, C. M., and Lodha, S. K. (1997). Approaches to uncertainty visualization. The Visual Computer, 13:370-390.

Pfaffelmoser, T., Reitinger, M., and Westermann, R. (2011). Visualizing the positional and geometrical variability of isosurfaces in uncertain scalar fields. In Computer Graphics Forum (Proceedings of EuroVis 2011).

Pizarro, O., Eustice, R. M., and Singh, H. (2009). Large area 3D reconstructions from underwater optical surveys. IEEE Journal of Oceanic Engineering.

Schmidt, G. S., ling Chen, S., Bryden, A. N., Livingston, M. A., Osborn, B. R., and Rosenblum, L. J. (2004). Multidimensional visual representations for underwater environmental uncertainty. IEEE Computer Graphics and Applications, 24:2004.

Thrun, S., Burgard, W., and Fox, D. (2005)). Probabilistic robotics. In MIT Press.

White, C., Hiranandani, D., Olstad, C., Buhagiar, K., Gabmin, T., and Clark, C. (2010). The Malta cistern mapping project: Underwater robot mapping and localization within ancient tunnel systems. In Journal of Field Robotics.

Williams, S., Newman, P., Dissanayake, G., and Durrant-Whyte, H. (2000)). Autonomous underwater simultaneous localization and map building. In Proceedings of the 2000 IEEE Int'l. Conference. 